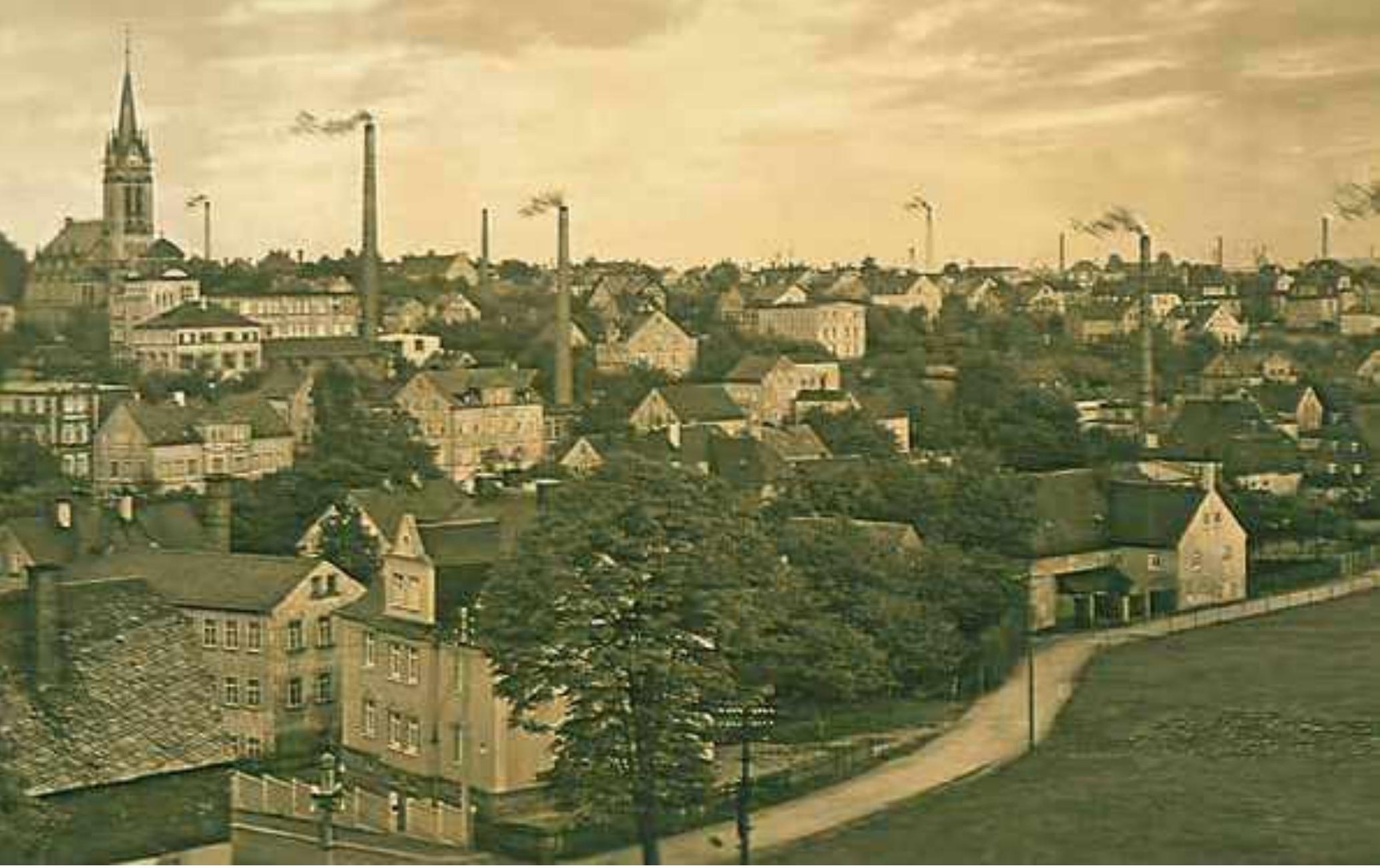

\title{
Oberfrohna und Limbach - Gemeinsamkeiten und Gegensätze
}

\author{
Hermann Schnurrbusch
}

Im Limbacher Land (Oberfrohna, Rußdorf, Kändler, Pleißa, Röhrsdorf, Bräunsdorf, Kaufungen, Mittel- und Niederfrohna, Fichtigsthal) des 19. Jahrhunderts gehörten etliche dieser Dörfer zum Rittergutsbezirk Limbach. Alle waren in Besiedlungswellen des 12. Jahrhunderts entstanden und hatten eine vergleichbare Entwicklung durchlaufen - vom feudalen, landwirtschaftlich geprägten Dorf zum bürgerlich gestalteten Industrieort. Die Zugehörigkeit von Rußdorf oder von Bräunsdorf und Kaufungen $\mathrm{zu}$ anderen Verwaltungsstrukturen und Obrigkeiten ist dabei eher zweitrangig.

Gemeinsamkeiten bestanden neben ihrer regionalen Lage im Vorerzgebirge und ihrer Mundart in der wirtschaftlichen, politischen und kulturellen Entwicklung. Alle profitierten nach der Einführung des ersten sächsischen Wirkstuhls durch Johann Esche um 1700 von der Wirtschaftsförderung der Limbacher Rittergutsher- rin von Schönberg, die Limbach zur „Wiege der sächsischen Wirkerei" machte und das Umland $\mathrm{zu}$ einem florierenden Industriebezirk. Hier entwickelten sich die regionalen Besonderheiten in der blühenden Strumpf-, Handschuhund Wirkwarenindustrie mit allen damit zusammenhängenden Bereichen des Textilmaschinenbaus, der Textilveredlung und den erfolgreichen Handelsbeziehungen in alle Welt. Als traditioneller Herrschaftssitz und durch Konzentration der Wirtschaftskraft, Anschluss an die Eisenbahn und Chausseen, Entwicklung der Bevölkerung und Infrastruktur bildete sich Limbach als Strukturzentrum dieses Raumes heraus, gefolgt von Oberfrohna. Nur diese zwei Dörfer schafften den Aufstieg vom Dorf zur Stadt: Limbach 1883, Oberfrohna 1935. Beide Städte strahlten auf das Umland aus mit Arbeitsplätzen, Kultur-, Bildungseinrichtungen (Zeitung, Kino) und anderen Faktoren der In-
Ansicht von Oberfrohna, 1943

๑ Stadtarchiv Limbach-Oberfrohna 
frastruktur. Die zentralistischen Bestrebungen Limbachs führten zum Gegensteuern des Umlandes gemeinsam mit Oberfrohna.

Oberfrohna war mit seinem Emanzipationsbestreben lange erfolgreich. Es bekam 1827 eine eigene Schule, 1886 das Postamt, 1889 Apotheke und Ärzte, 1853 den ersten und 1890 den zweiten Friedhof, 1893 eine eigene Kirche und Kirchgemeinde. Alle diese Obliegenheiten hatte vorher Limbach für Oberfrohna wahrgenommen. Durch eine Blüte der Handschuhindustrie um 1900 und der Wirkwarenfabrikation in den 1930er Jahren, Bevölkerungszuwachs und Entwicklung der Infrastruktur (Gas-, Elektrizitäts-, Wasserwerk) erstarkte Oberfrohna so, dass es am 1. April 1935 Rußdorf eingemeinden und mit über 10.000 Einwohnern zum 1. Juni 1935 die Bezeichnung Stadt erhalten konnte. Oberfrohna wurde wohl zu Recht in der Presse als die „blühendste der drei Frohnen“ bezeichnet. Tatsächlich war Oberfrohna finanziell besser gestellt als seine Nachbarn (Limbach kam 1932 wegen des riesigen Defizits unter Zwangsverwaltung), hatte eigene Versorgungseinrichtungen, höhere Steuereinnahmen, bessere Straßen usw. Limbach argumentierte mit Berufsschule, Museum, Kinos, Amtsgericht, Wannenbad. Den Forderungen nach Expansion der KPD im „roten Limbach" trat nach 1933 die NSDAP auch dadurch entgegen, dass sie neben Rußdorf und Oberfrohna ebenso Niederfrohna mit Mittelfrohna und Fichtigsthal vereinte. Das geschah am 1. Januar 1936.

Limbacher Expansionsversuche haben eine lange Vorgeschichte. Arno Fritzsch (SPD), besoldeter Stadtrat (Dezernent) in Limbach, plante schon seit 1919 ein „Großlimbach“. Dazu sollte Limbach die Orte Oberfrohna, Mittelfrohna, Rußdorf, Pleißa und Kändler eingemeinden und so mit etwa 40.000 Einwohnern eine Großgemeinde bilden. An die Eingliederung von Wolkenburg, Dürrengerbisdorf oder Uhlsdorf hätte Fritzsch wohl in seinen kühnsten Träumen nicht gedacht. Sein Plan ist gescheitert. Ende der 1920er Jahre forderte die Limbacher KPD wieder die Bildung von „Großlimbach“. Sie wollte mit der KPD der umliegenden Dörfer in Limbach zu einer Mehrheit kommen. Aber die Bürgermeister der Dörfer stellten sich dagegen, selbst der KPD-Bürgermeister von Mittelfrohna, der daraufhin aus seiner Partei ausgestoßen wurde.

Mittelfrohna stellte 1931 noch einmal den Antrag auf „Einverleibung durch Limbach“. Das lehnten die Limbacher 1932 wegen der Überschuldung Mittelfrohnas ab. Nach Verhandlungen seit 1852 kam die Angliederung des Mittelfrohnaer Ortsteils Kreuzeiche an Limbach am 1. April 1931 zustande. Zu diesem Anlass hatten die Behörden beider Orte die Einwohner zu einem Fest mit Feuerwerk in den „Gasthof Kreu- zeiche“ eingeladen. Die meisten kamen aber nicht, weil sie die Ankündigung für einen Aprilscherz des „Limbacher Tageblattes“ hielten, dem sie einige Jahre zuvor schon einmal aufgesessen waren.

Nach 1945 ergaben sich neue Verhältnisse. Als erstes versuchte die Rußdorfer KPD, ihren Ort von Oberfrohna zu lösen und wieder selbständig zu machen - ohne Erfolg. Am 18. November 1946 forderte der Limbacher Stadtrat vom Landrat die Einverleibung Oberfrohnas. Die Oberfrohnaer lehnten dies einmütig ab. Einen erneuten Limbacher Antrag, eine „Großgemeinde Limbach“ zu bilden, wies der Kreistag am 7. Mai 1947 ab. Im März 1949 gab es auf Betreiben der Stadt Limbach wieder Bestrebungen, „das gesamte Wirtschaftsgebiet zusammenzuschweißen“. Limbach wollte die Vereinigung mit Oberfrohna, Niederfrohna, Pleißa, Kändler und Bräunsdorf zu einer Stadt mit 40.000 Einwohnern. Die schwierigen Nachkriegsverhältnisse und die Bedingung des freiwilligen Zusammenschlusses ließen auch dieses Projekt scheitern.

1950 kam die Anordnung von oben. Die Landesregierung Sachsen beschloss mit Gesetz vom 27. April 1950 Eingemeindungen in Sachsen, so auch die Vereinigung der Städte Limbach und Oberfrohna. ${ }^{1}$ Durch dieses Gesetz wurden in unserer näheren Umgebung am 1. Juli 1950 auch Adelsberg, Erfenschlag, Glösa, Harthau, Rabenstein sowie die Stadt SiegmarSchönau von Chemnitz einverleibt. Es handelte sich um eine Entscheidung des Landes zur Strukturentwicklung in Sachsen, bei der die eingemeindeten Orte keine Chance zur Gegenwehr hatten. Vermutlich spielte auch die späte Rache der an die Macht gekommenen Limbacher Kommunisten gegenüber dem renitenten Nachbarn eine Rolle. Oberfrohna war im Gegensatz zu Limbach, bis 1933 eine kommunistischen Hochburg, eher bürgerlich konservativ geprägt. Vielleicht lag das auch an der in mancher Hinsicht paternalistischen Haltung der Fabrikanten. Theodor Grobe war dafür bekannt, dass er seinen Arbeitern auskömmliche Löhne und erträgliche Arbeitsbedingungen zugestand, aber jeden entließ, der sich kommunistisch betätigte. Während sich in Limbach Streiks wiederholten, kamen sie in Oberfrohna kaum vor.

Die „Volksstimme“ vom 3. April 1950 berichtete über eine Arbeitskonferenz des Kreisrates über die geplante Zusammenlegung der Städte Limbach und Oberfrohna. Der Bürgermeister Selkmann (SED, vorher SPD) von Oberfrohna sprach sich für die Vereinigung aus, wollte aber eine Reihe von Problemen vorher gelöst wissen. Der Limbacher Bürgermeister Fischer (SED) betonte die Vorteile des Zusammenschlusses in wirtschaftlicher, kultureller und politischer 
Hinsicht. Die Stadtverordneten in Limbach stimmten dem Vorhaben zu.

Eingemeindungen sind aus Sicht der Strukturentwicklung eines Landes notwendig und unausweichlich. Über die Jahrhunderte entwickelt sich eine Region nicht gleichmäßig, es entstehen immer zentrale Strukturen: Burgen, Märkte, Verkehrsknoten, Wirtschaftszentren, Städte. Aus manchen Dörfern werden Städte, aus einigen Städten Großstädte. Nur das Herausbilden von Zentren führt auch zur besseren Versorgung des Umlandes. Man kann nicht einen Verwaltungssitz, eine Universität oder eine große Klinik, Theater und Einkaufszentren auf dem Dorf errichten (wie in Hartmannsdorf oder Röhrsdorf), ohne die zentralen Versorgungsfunktionen zu beeinträchtigen. Natürlich lässt sich in Wolkenburg eine Philharmonie installieren oder im Limbach-Oberfrohnaer Stadtpark ein Zoo, aber solche Vorhaben entsprechen nicht der allgemeinen Strukturentwicklung. Zentren vergrößern sich, wachsen mit Nachbargemeinden zusammen, brauchen aber zum Erfüllen ihrer Funktion auch den Zuwachs an Einwohnern, Kaufkraft, Flächen, Steuern. Es kommt also - auch aus Gründen effizienter Verwaltung - immer wieder zu Eingemeindungen wie um 1900, um 1935, 1950 und noch jetzt. 1990 gab es in Sachsen 1.626 Gemeinden, davon 170 Städte (6 kreisfreie) und 48 Kreise. Jetzt im Jahre 2016 gibt es noch 426 Gemeinden, davon 170 Städte.

In Oberfrohna überwogen bei einer Einwohnerversammlung am 24. Mai 1950 Ablehnung und Kritik die SED-konforme Zustimmung zur Frage des Zusammenschlusses. „Aus blindem Ortspatriotismus, Eigenbrötelei, Überheblichkeit, Intrige, Rückschritt“, meinte das SED-Blatt „Volksstimme“ am 10. Juni 1950. Tatsächlich war die Mehrheit der Oberfrohnaer dagegen, durchaus begründet, wie die Entwicklung ihres Ortsteils in den kommenden Jahren zeigen sollte. Dessen ungeachtet stimmte die Stadtverordnetenversammlung von Oberfrohna am 25. Mai 1950 mit der Mehrheit von SED und LDP der „Zusammenlegung“ zu. Charakter zeigte lediglich die CDU-Fraktion mit dem Stadtrat Zwingenberger, sie enthielt sich der Stimme. Zwingenberger wurde daraufhin unter Druck gesetzt und legte sein Mandat nieder. Danach spurte auch die CDU nach SED-Kommando.

Beide Städte bildeten eine „Kommission betreffs Zusammenlegung“ und legten organisatorische Schritte fest: Auflösung der Stadtverwaltung Oberfrohna, Erfassung der Vermögenswerte und finanziellen Verpflichtungen und die Struktur der Verwaltung für die neue Stadt. Die neuen Stadtgebiete sollten die Ziffern I für Limbach, II für Oberfrohna und III für Rußdorf bekommen, Stadtfarben, Wappen und Siegel von Limbach sollten für die neue Stadt gelten. Nach
Abschluss dieser Planungen beschloss die Kommission am 16. Juni 1950 einstimmig die Zusammenlegung. Das Gesetzblatt vom 27. Juni 1950 verkündete die Zusammenlegungen im Landkreis Chemnitz, so auch von Limbach und Oberfrohna mit dem neuen Gemeindenamen „Limbach-Oberfrohna“.2

Nun also Limbach-Oberfrohna. Über den Namen der neuen Stadt hatte es erbitterte Auseinandersetzungen gegeben. Sowohl Limbach als auch Oberfrohna machten - zu Recht - geltend, ihr Ortsname sei weltbekannt, weil mit Hunderten Industrieprodukten verbunden. Keiner wollte auf den guten Klang seines Städtenamens verzichten. Die Oberfrohnaer argumentierten damit, dass ihr Name in Deutschland einmalig sei, Limbach hingegen fünfzehn Mal vorkäme. Gegen den Vorbehalt, dass ein Doppelname zu lang wäre, einigte man sich dann doch auf Limbach-Oberfrohna. Der Name hat sich bis heute noch nicht gänzlich durchgesetzt. Faulheit oder Denkschwäche spricht und schreibt oft von Limbach, wenn die ganze Stadt gemeint ist. Jeder Informierte fragt, wenn er Limbach hört, welches? Denn es gibt mehrere. Befremdlich ist der in der Presse genannte „Limbacher“, der in Oberfrohna wohnt.

Am 30. Juni 1950 trafen sich die Stadtverordneten von Limbach und Oberfrohna zur gemeinsamen Sitzung im Jahnhaus. Es war die letzte Versammlung der Oberfrohnaer Vertreter. Sie hatten vorher schnell noch 6.000 Mark der Betriebssportgemeinschaft Industrie (BSG) für ihre Sportanlage am Jahnhaus zugewendet und 750 Mark der Gerhart-Hauptmann-Schule. Das letzte verfügbare Geld sollte Limbach nicht bekommen. Beide Kollegien bejahten nun einstimmig den Zusammenschluss. Unter kommunistischem Regime endeten Abstimmungen immer einstimmig. Bürgermeister der Stadt wurde das Limbacher Stadtoberhaupt Herbert Fischer (SED, Stasi-Mitarbeiter), im Amt vom 1. Januar bis 17. September 1950. Der seit 13. Juli 1946 amtierende Oberfrohnaer Bürgermeister Carl Selkmann (SED) wurde besoldetes Ratsmitglied der neuen Stadtverwaltung. Beide bekundeten wie die Sprecher der Parteien „einmütig die Freude über die Vereinigung und den Willen zur Zusammenarbeit“. ${ }^{3}$ Alle Parteien gaben ihr Statement ab und sprachen von ,geschichtlichem Wendepunkt“ (LDP), „Realisierung längst gehegter Wünsche“ (CDU) und „Stärkung unserer antifaschistisch-demokratischen Ordnung“ (SED). Oft war die Rede von friedlicher Zukunft, Weltfrieden und dem „Besten der Stadt“. Im Herbst fanden sowieso Neuwahlen statt, diesmal mit der Einheitsliste.

Damit war die Eingemeindung perfekt. Zu dieser Zeit hatte Limbach 17.593 und Oberfrohna 11.078 Einwohner, zusammen jetzt 28.671. Die
2 Gesetzblatt Nr. 72 vom 27. Juni 1950, S. 472.

3 Volksstimme vom 6. Juli 1950. 
Rosen-Apotheke und Umgebung, 1993. Das Gebäude wurde 2004 abgebrochen. Foto: Hermann Schnurrbusch

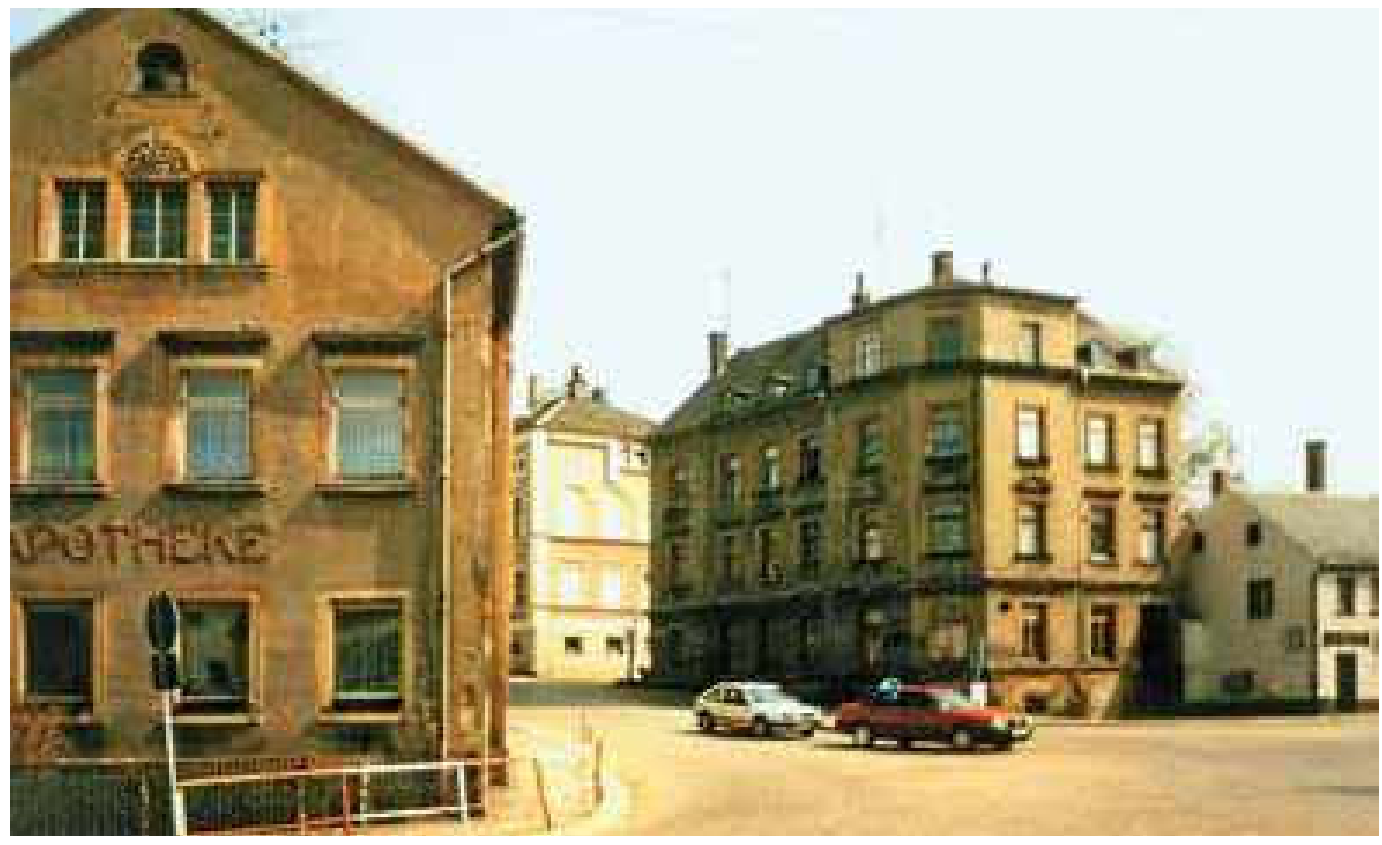

großen Zahlen kamen durch die vielen Neubürger (Flüchtlinge, Vertriebene, Heimkehrer seit 1945) zustande, in Limbach etwa 4.000 , in Oberfrohna rund 2.000. Die neue Stadt umfasste nun $15,7 \mathrm{~km}^{2}$; von Limbach $8,8 \mathrm{~km}^{2}$ und $6,9 \mathrm{~km}^{2}$ von Oberfrohna. Im Jahre 2015 hat die Große Kreisstadt Limbach-Oberfrohna sieben Ortsteile, rund $50 \mathrm{~km}^{2}$ Fläche und 24.406 Einwohner. Also nun doch noch „Großlimbach“, wie seit 70 Jahren beabsichtigt. Die Zusammenlegung führte zu Doppelungen von Straßennamen, es wurden Umbenennungen notwendig. Als ein Symbol der Einigkeit wurde gepriesen, dass aus der Frohnaer Straße in Limbach und der Limbacher Straße in Ober- frohna nun die einheitliche „Straße des Friedens" wurde, die leider heute immer noch so heißt, weil sich keiner über den kommunistisch-propagandistischen Hintergrund des $\mathrm{Na}-$ mens Gedanken macht.

Volksmund, beim Friseur erzählt: „In der Geschichte von Oberfrohna gab es zwei Unglückstage: Den einen, als 1945 die Amerikaner abzogen und die Russen kamen und den anderen, als Oberfrohna 1950 von Limbach eingemeindet wurde."

Die Mangelwirtschaft der DDR und die Benachteiligung durch die Kommunisten bewirken den Niedergang des Ortsteils Oberfrohna. Läden schlossen, Gebäude verfielen, Men-
Noch immer sind in Oberfrohna zahlreiche Brachen zu sehen. Hier der leerstehende Fabrikstandort der Firma Schulze \&t Grobe, 2005 Foto: Hermann Schnurrbusch

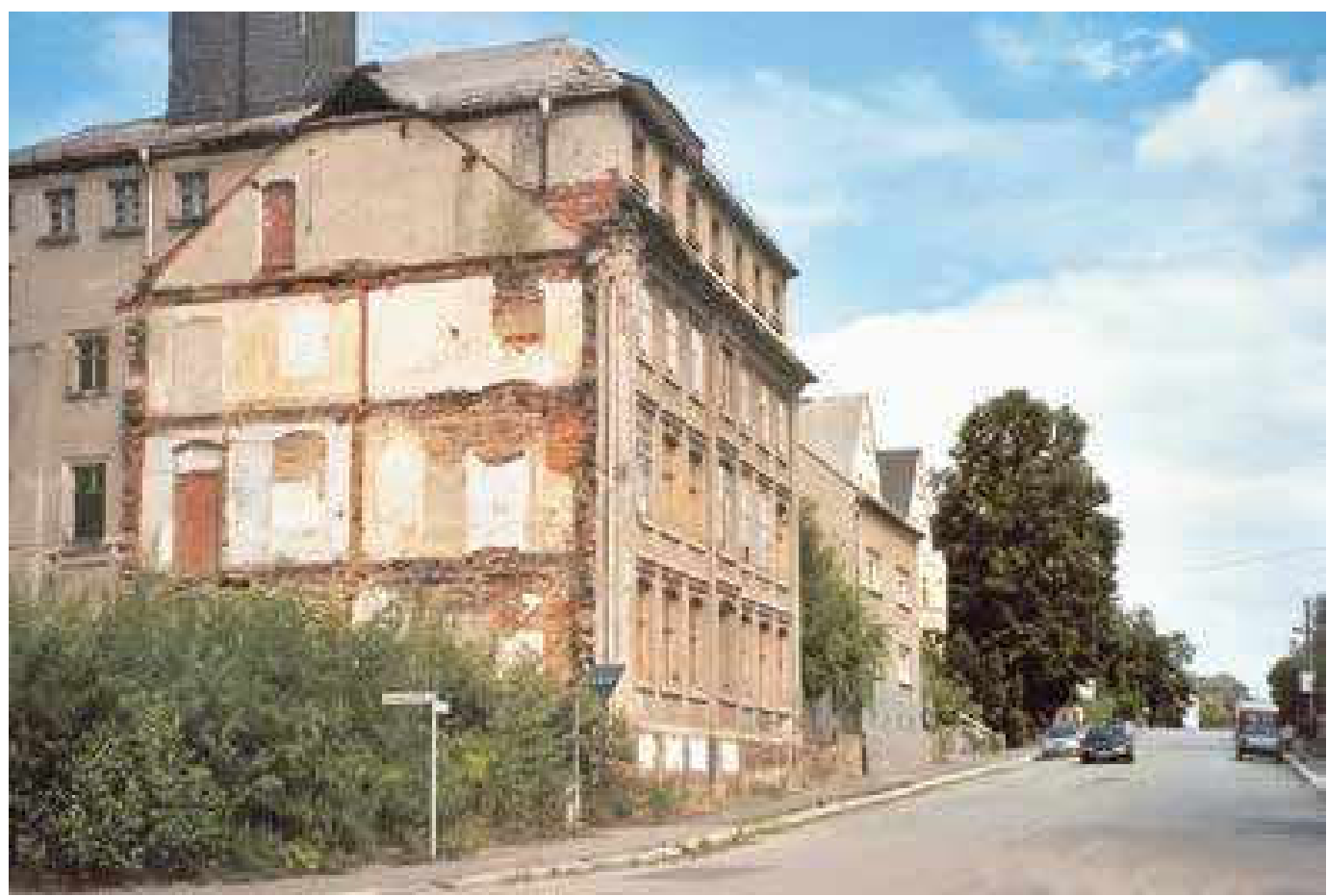




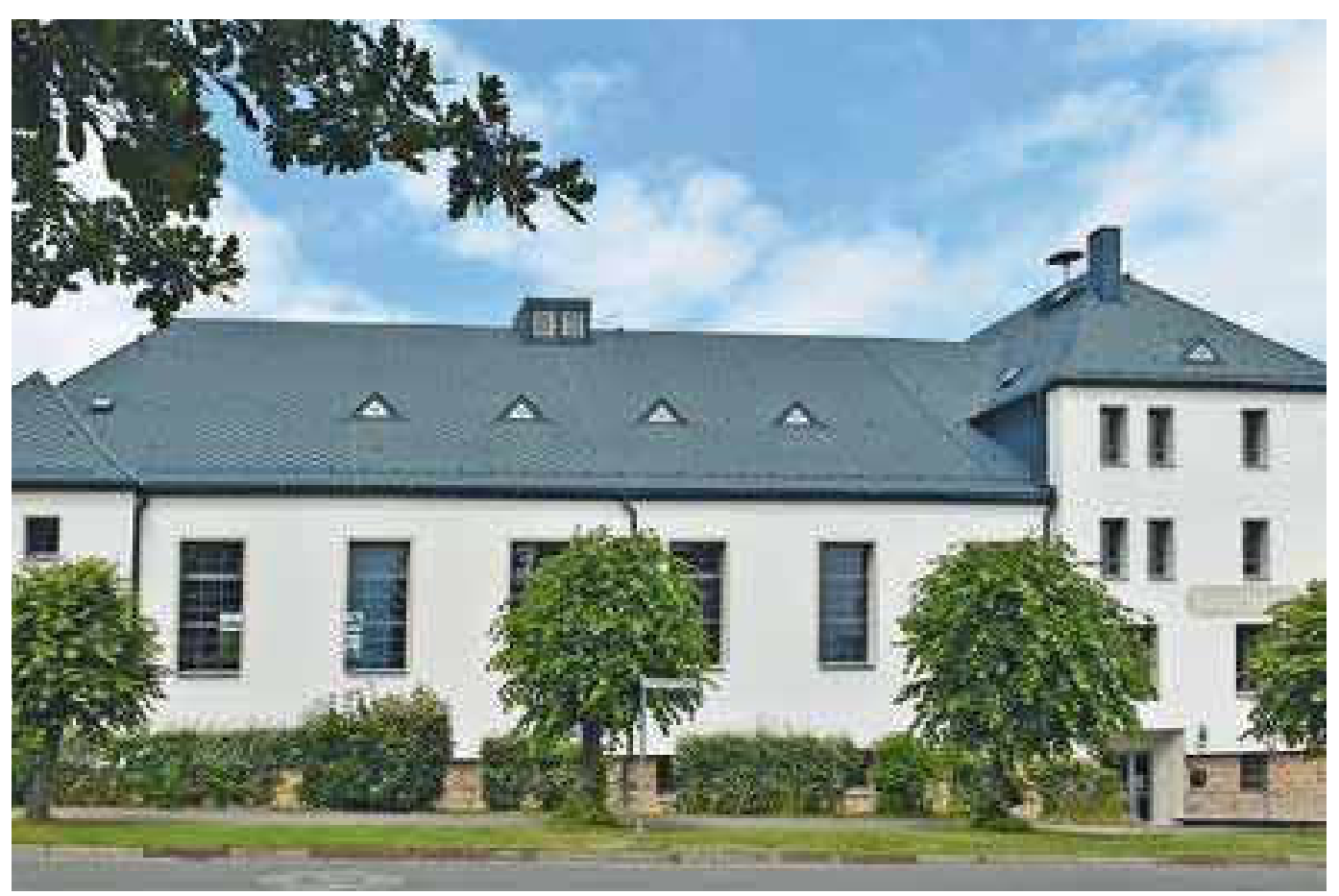

schen flohen westwärts. Wenn Neubaugebiete entstanden, dann in Limbach an der Straße der Genossenschaft, Am Hohen Hain oder Wasserturm. Für Oberfrohna reicht die „Baukapazität“ für den „Pappelhain“, aber nicht, um den Verfall aufzuhalten.

1990 kam mit der deutschen Wiedervereinigung erneut Hoffnung auf, den verfallenden Stadtteil wiederbeleben zu können. Aber auch diesmal reicht es nicht für Oberfrohna. Man könnte meinen, die Entwicklung der Stadt Limbach-Oberfrohna finde in Limbach statt, Oberfrohna bleibe das fünfte Rad am Wagen. Wohnungsbau auf der Süd-, Teichstraße oder Marktsteig, Geschäftshäuser an Jäger- und Weststraße oder am Johannisplatz, die Innenstadtsanierung oder Vorhaben wie Sporthalle, „Spaßbad“ oder „Amerika-Zoo“ gibt es in Limbach, nichts Vergleichbares im Ortsteil Oberfrohna, nicht einmal einen Kinderspielplatz. Selbst 2015 zielt die Stadtentwicklung auf die „Innenstadt“ - Helenenstraße, Johannisplatz. In Oberfrohna gibt es in vielen Straßen Ruinen und Brachen, nicht nur von Fabriken.

Heute gibt es bei Eingemeindungen Bedenken, die Identität des bisherigen Dorfes ginge verloren, der ländliche Charakter, der vertraute Name. Natürlich können sich Einwohner über die Jahrhunderte mit ihrem kleineren Ort viel besser identifizieren, als das mit einer größeren, vielleicht sogar als feindlich empfundenen Stadt geschehen kann. Dort hilft nur viel Geduld, aber es gibt auch nach mehreren Generationen immer noch „Rußdorfer“. Wichtiger scheint, dass die örtliche Gemeinschaft - ob als Dorf oder als Stadtteil - funktioniert. Solange die Einwohner miteinander kommuni- zieren, sich in Vereinen begegnen, verbunden fühlen und zusammen Feste feiern, ist es nicht so wichtig, wie die Gemeinschaft heißt. Positive dorf.

Leider kann auch jahrzehntelange Unterdrückung und Benachteiligung, Mangelwirtschaft und Verfall von Kristallisationspunkten der Kommunikation, der Wegzug junger Leute, der Wegfall von Arbeitsmöglichkeiten die örtliche Gemeinschaft kaputtmachen. Oberfrohna ist auf einem solchen Tiefpunkt, zumal die Kommunalpolitik seit 1990 ein Ortschaftszentrum des geselligen und gesellschaftlichen Lebens und eine Verbesserung der maroden Versorgungs- und Infrastruktur nicht schaffen konnte oder wollte. Nicht einmal ein Ortschaftsrat mit einem Ortsvorsteher vertritt die Interessen dieses Ortsteils. Oberfrohna hat nicht die entsprechende Lobby.

Die Oberfrohnaer werden ihr über 100 Jahre altes Bemühen um Gleichberechtigung fortsetzen müssen oder zweitrangiger, verfallender Ortsteil bleiben. Es ist unerheblich, ob in Limbach der Feudalherr oder die Politik-Lobby einer Partei sitzt. Auch unter demokratischen Verhältnissen hat nur der eine Chance, der sich durchsetzt. Hoffen wir, dass sich die Bürger dieses Stadtteiles an ihre Geschichte erinnern und an die Tatsache, dass jeder nur das erreicht, was er selbst erkämpft. Allerdings müssten die Bewohner von Oberfrohna sich ihrem Ortsteil zugehörig und verantwortlich fühlen und wieder zu einer Gemeinschaft finden, wie sie in Bräunsdorf, Pleißa, Rußdorf u. a. noch vorhanden ist. Gibt es noch Oberfrohnaer? Beispiele dafür sind Rußdorf oder Bräuns-
Das in den 1920er Jahren errichtete Jahnhaus wurde 2014 saniert. Mit kleinem Turnsaal, Turnhalle und Außensportanlagen wird es von Schule und Vereinen rege genutzt.

\section{Autor}

Dr. Hermann Schnurrbusch Limbach-Oberfrohna 